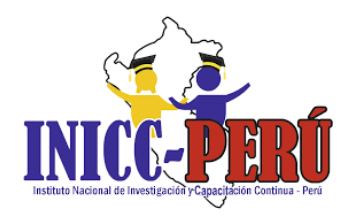

\title{
La formación investigativa en los estudiantes de licenciatura en Educación Básica. Una revisión sistemática
}

\author{
Research Training for Undergraduate Students in the Elementary Education \\ Bachelor's Program. A Systematic Review
}

\section{MIGUEL EDMUNDO NARANJO-TORO}

https://orcid.org/0000-0001-6521-1405

\section{FRANK EDISON GUERRA-REYES ${ }^{2}$}

https://orcid.org/0000-0003-3253-6419

\begin{abstract}
RESUMEN: Emprender procesos para la implementación de la investigación formativa, constituye en la actualidad un requerimiento ineludible que deben afrontar las Instituciones de Educación Superior. El presente artículo, propone una revisión sistemática relacionada con la investigación formativa implementada con estudiantes que se forman como docentes. El propósito fundamental de este estudio fue sistematizar las principales fuentes documentales relacionadas con la investigación formativa en estudiantes universitarios de las carreras de Educación Básica. El estudio encontró que la investigación formativa, como guía para la enseñanza-aprendizaje, constituye una respuesta académica y de solución a los problemas de la vida social y profesional de los educandos en formación y de la comunidad en donde se insertan. Asimismo, posibilita, tanto a estudiantes como docentes, motivación por la actividad investigativa, reflexión sobre normas éticas, preparación en conocimientos, metodologías y desarrollo de procesos cognitivos superiores y de investigación guiada.
\end{abstract}

Palabras clave: investigación formativa; formación docente; educación básica; revisión sistemática.

\begin{abstract}
To undertake processes for the implementation of training research is currently an unavoidable requirement that must be faced by Higher Education Institutions. The present article proposes a systematic review related to the formative research implemented with students who are trained as teachers. The main purpose of this study was to systematise the main documentary sources related to training research on university students in Basic Education. The study found that formative research, as a guide to teachinglearning, constitutes an academic response and solution to the problems of the social and professional life of learners in training and the community in which they are inserted. Likewise, it enables both students and teachers to be motivated by research activity, to reflect on ethical standards, to be prepared in knowledge, methodologies and to develop higher cognitive processes and guided research.
\end{abstract}


Keywords: Formative research; teacher training; elementary education; systematic review.

\section{INTRODUCCIÓN}

La primacía del modelo tradicionalista de la enseñanza ha sido superada por las exigencias de una sociedad globalizada y por los cambios sustanciales en la forma de aprender en la sociedad digital. Más allá de un modelo didáctico reproductivo, que no ha logrado el desarrollo integral de procesos investigativos, la sociedad contemporánea necesita preparar a las nuevas generaciones en modelos didácticos productivos que les habiliten para la construcción y producción de nuevos aprendizajes.

Múltiples investigaciones, realizadas a nivel mundial, han demostrado que la función emblemática de las universidades es la generación y difusión de conocimiento. Las instituciones de educación superior existen para tratar de descubrir nuevos conocimientos que permitan comprender los fenómenos naturales, sociales y humanos; $\mathrm{y}$, con ello incidir en su desarrollo. Es decir, la función investigativa de la academia debería estar orientada a comprender y solucionar los dilemas y necesidades del contexto en la que está inserta.

No obstante, en el contexto ecuatoriano, en particular, son insuficientes las propuestas de desarrollo investigativo dirigidas para los estudiantes que se sustenten en la enseñanza para la comprensión, el desarrollo de procesos investigativos y de pensamiento crítico, así como el uso pertinente de las nuevas tecnologías de información y la comunicación.

De cara a esta exigencia para el mejoramiento de los procesos investigativos, en este manuscrito se presentan algunas conclusiones relacionadas con la relevancia científica de la investigación formativa, que han asignado múltiples estudios realizados en Hispanoamérica, Brasil y Estados Unidos. (Blessinger y Carfora, 2014; Hernández et al, 2011; Oropeza, 2016; Ronquillo, 2017; Ruíz del Castillo y Rojas-Soriano, 2006; y, Torres, s.f.). Por otra parte, el proceso metodológico se realizó con base en las propuestas de: Aguilera-Morales et al, 2018; Gómez-Luna, Fernando-Navas, Aponte-Mayor y Betancourt-Buitrago, 2014; Gough, Oliver y Thomas, 2012; Torres-Verdugo, 2016; y, UNAM, 2015.

La búsqueda de información en la literatura especializada fue fundamental para estudiar los aspectos relacionados con la investigación formativa y sus referentes. Por ello, en primer lugar, se planteó una pregunta que guie las búsquedas, para seguidamente, determinar las palabras relacionadas con la revisión sistemática, a saber: ¿Cuáles son las principales fuentes documentales relacionadas con la investigación formativa con estudiantes universitarios de las carreras de educación básica?

\section{METODOLOGÍA}

Los términos de búsqueda empleados fueron "investigación formativa" or "formative research" or "inquiry-based learning"; "investigación en aula" or "aprendizaje basado en la indagación"; "higher education", or "college" or "university". Para encontrar la información que sustenta este estudio se revisaron documentos académicos y fuentes de información, entre las que se priorizaron: reportes técnicos, artículos científicos, tesis y libros, ubicados en 
bases de datos, buscadores, plataformas, repositorios digitales y bibliotecas universitarias, tal como se muestra en la tabla 1:

Tabla 1

Cuadro de resumen sobre fuentes consultadas

\begin{tabular}{lllll}
\hline Buscadores & $\begin{array}{c}\text { Bases de } \\
\text { datos }\end{array}$ & $\begin{array}{l}\text { Plataformas de } \\
\text { Empresas editoriales }\end{array}$ & Repositorios digitales & Bibliotecas \\
\hline BASE & Dialnet & $\begin{array}{l}\text { Taylor \& Francis } \\
\text { Online }\end{array}$ & $\begin{array}{l}\text { Red de repositorios } \\
\text { de acceso abierto del } \\
\text { Ecuador } \\
\text { Repositorio digital }\end{array}$ & Biblioteca UTN \\
Dimensions & ProQuest & Springer Link & $\begin{array}{l}\text { BTN } \\
\text { Consorcio de } \\
\text { bibliotecas } \\
\text { universitarias del } \\
\text { Ecuador }\end{array}$ & \\
DOAJ & Redalyc & & & \\
Google & SciELO & & & \\
Académico & La & & & \\
Referencia & Scopus & & \\
\hline
\end{tabular}

Fuente: Base de datos de acceso para los investigadores

Tipo de estudios seleccionados: se realizó una búsqueda de investigaciones en español, portugués e inglés, de tipo: descriptivas, cualitativas y cuantitativas.

Organización y síntesis de la información: en primer lugar, se determinó que la investigación formativa constituye un objeto de estudio de actualidad, tal como se evidencia en el diagrama generado en la base de datos Scopus (figura 1).

Figura 1

Artículos científicos publicados sobre investigación formativa en los últimos diez años

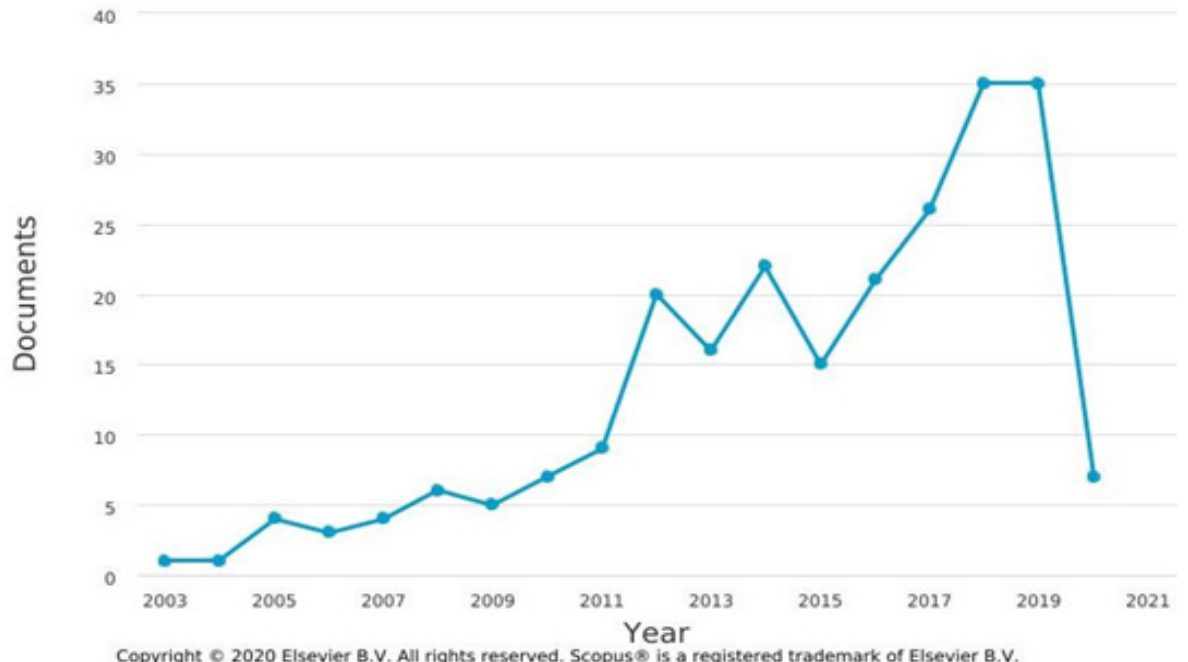

Fuente: Base de datos de los investigadores 


\section{Proceso de identificación de artículos}

1. Fase de identificación

En una aproximación al tema de estudio se ubicaron 408 resultados a través de la búsqueda sistemática. En las figuras 2 y 3, se muestra un ejemplo de la tabla de sintaxis de dos bases de datos: Scielo y Scopus, a saber:

Figura 2

Tabla de sintaxis SciELO

22 documentos encontrados: (investigación formativa en la universidad) AND in:("scl" OR "mex" OR "col" OR “chl” OR "arg" OR “ecu”) AND la:(“es" OR "pt") AND year_cluster:(“2018” OR "2016" OR “2017” OR “2019” OR “2020”) AND type:(“research-article”) AND subject_area:("Human Sciences” OR "Applied Social Sciences")

Fuente: Base de datos SciELO

Figura 3

Tabla de sintaxis Scopus

218 document results

TÍTULO-ABS-KEY-AUTH ( formative research ) Y ( LIMITE DE-A ( PUBSTAGE , "final" ) ) Y ( LIMITE DE-A ( accessType (OA) ) ) Y ( LIMITE DE-A ( AFFILCOUNTRY, "Estados Unidos" ) O LIMIT-TO ( AFFILCOUNTRY , "Reino Unido" ) O LIMIT-TO ( AFFILCOUNTRY , "España") O LIMIT-TO (

AFFILCOUNTRY , "Brasil" ) O LIMIT-TO ( AFFILCOUNTRY , "Colombia" ) O LIMIT-TO ( AFFILCOUNTRY , "Mexico" ) O LIMIT-TO ( AFFILCOUNTRY , "Chile" ) O LIMIT-TO ( AFFILCOUNTRY , "Argentina" ) O LIMIT-TO ( AFFILCOUNTRY, "Cuba") O LIMIT-TO ( AFFILCOUNTRY , "Peru") O LIMIT-TO ( AFFILCOUNTRY , "Ecuador" ) O LIMIT-TO ( AFFILCOUNTRY , "Puerto Rico" ) O LIMIT-TO ( AFFILCOUNTRY , "Venezuela" ) O LIMIT-TO ( AFFILCOUNTRY , "Costa Rica” ) O LIMIT-TO ( AFFILCOUNTRY , “República Dominicana “ ) O LIMIT-TO ( AFFILCOUNTRY , " Haití “ ) O LIMIT-TO ( AFFILCOUNTRY , " Jamaica “) O LIMIT-TO ( AFFILCOUNTRY , "Panamá" ) O LIMIT-TO ( AFFILCOUNTRY , "Uruguay" ) O LIMIT-TO ( AFFILCOUNTRY , "Bolivia" ) O LIMIT-TO ( AFFILCOUNTRY , "El Salvador" ) O LIMIT-TO ( AFFILCOUNTRY , "Honduras") O LIMIT-TO ( AFFILCOUNTRY, "Nicaragua" ) ) Y ( LIMIT-TO ( PUBYEAR, 2020 ) O LIMIT-TO ( PUBYEAR, 2019 ) O LIMIT-TO ( PUBYEAR , 2018 ) O LIMIT-TO ( PUBYEAR , 2017 ) O LIMIT-TO ( PUBYEAR , 2016 ) ) Y ( LIMIT-TO ( DOCTYPE , “ar “) ) Y ( LIMIT-TO ( SUBJAREA , “ SOCI “ ) O LIMIT-TO ( SUBJAREA , “ARTES” ) ) Y ( LIMIT-TO ( IDIOMA , "Inglés" ) O LIMIT-TO ( IDIOMA , "Español” ) O LIMIT-TO ( IDIOMA , "Portugués") )

Fuente: Base de datos Scopus

2. Fase de selección

Se eliminaron los artículos que no presentaban las palabras claves: "investigación formativa" or "formative research" or "inquiry-based learning" or "investigación en aula" or "aprendizaje basado en la indagación" or "higher education" or "college" or "university", en el título y/o resumen. 
3. Fase de inclusión (establecer criterios de inclusión y exclusión)

Se incluyeron en la actual indagación, los artículos que cumplieron con los siguientes criterios: investigación descriptiva, cualitativa y cuantitativa, en español, inglés o portugués, muestra de estudiantes universitarios de pregrado y el que el tema medular sea la Investigación Formativa. Se excluyeron los estudios con muestra distinta a estudiantes universitarios de pregrado y postgrado.

4. Fase de sesgo

Se contó con la participación de dos docentes investigadores que analizaron el proceso de búsqueda y selección de los estudios y el proceso de análisis de los estudios incluidos. Estos revisores realizaron sus análisis en forma independiente entre sí.

5. Proceso de análisis de información

Luego de la ejecución de las búsquedas en las variadas fuentes de información académicas, antes referidas, se determinaron 76 documentos.

a) Tema planteado: Investigación formativa en el nivel superior

Con relación a la investigación formativa, existen 09 estudios realizados a nivel nacional, en Ecuador:

b) Repositorio digital Universidad Técnica del Norte. Ecuador

- Un trabajo de pregrado: Consiste en una investigación descriptiva que usa como instrumento para la recolección de datos un cuestionario. Al final, los resultados se analizan a partir de la opinión de los estudiantes informantes. Por lo tanto, la posibilidad de generalizar los hallazgos es limitada.

c) Red de repositorios de acceso abierto del Ecuador (RRAAE) y Consorcio de Bibliotecas Universitarias del Ecuador (COBUEC)

- Cuatro tesis de maestría: En estos trabajos investigativos se determinan los resultados de los procesos enseñanza-aprendizaje sustentados en la investigación formativa. Aunque luego de su revisión se determinó que contienen limitaciones en sus procedimientos investigativos, en tanto, la mayoría de ellos no usa de manera adecuada la metodología investigativa.

- Cuatro artículos científicos: Ejemplos de cómo esta metodología posibilitó el desarrollo investigativo en el nivel de posgrado. Estos documentos se encuentran referenciadas en repositorios digitales de acceso abierto y plantean una perspectiva básica relacionada con la situación y los proyectos de investigación formativa implementados en cuatro universidades ecuatorianas.

d) Bases de datos bibliográficas UTN

Con relación a la investigación formativa, se encontraron 67 estudios realizados a nivel internacional, de los cuales 61 corresponden a artículos científicos, tres tesis de doctorado y tres libros: 
6. Artículos científicos:

- Scopus. Ocho artículos

- SciELO. Diez artículos

- Dialnet. Un artículo

- DOAJ. 30 artículos

- Redalyc. 12 artículos

7. tres tesis de doctorado:

8. Tres libros:

e) Reflexión sobre los sitios y herramientas web

Es importante resaltar las ventajas que, para los procesos investigativos, se generan al contar con una amplia disponibilidad de las bases de datos académicas, antes enunciadas. Sin embargo, para el objeto de investigación planteado, resultan con mayor utilidad las bases de datos asequibles y de pago de la Universidad, a saber: Scopus, SciELO y Dialnet. Por otra parte, también son de alto nivel académico aquellas de libre acceso como: La Referencia, DOAJ y Redalyc.

\section{RESULTADOS}

Luego de la ejecución del anterior proceso se enlistan los 76 documentos seleccionados, a saber:

a) Un trabajo de pregrado:

- Análisis de la investigación formativa y su relación con el desarrollo de destrezas de los estudiantes del sexto semestre de la carrera de Biología Química y Laboratorio en la Universidad Nacional de Chimborazo octubre 2015-febrero 2016-Fuela Pérez María Belén-Universidad Nacional de Chimborazo-Escuela de Biología, Química y Laboratorio- 2016.

b) Cuatro tesis de maestría:

- Estrategias Didácticas de investigación formativa que permitan incentivar el desarrollo de proyectos de investigación a los estudiantes de la Facultad de Administración Finanzas e Informática de la Universidad Técnica de Babahoyo-Gonzáles Valero, María Isabel-Universidad Técnica de Babahoyo-Maestría en docencia y curriculo-2013.

- La investigación formativa aplicada como metodología didáctica para el aprendizaje de parasitología en los estudiantes de séptimo semestre de la Carrera de Laboratorio Clínico de la UNACH. periodo mayo-noviembre 2016-Balladares Saltos, Aida Mercedes-Universidad Nacional de Chimborazo- Maestría en ciencias de la educación, mención biología-2017.

- Sistema de acciones para potenciar la investigación formativa en el Colegio Técnico Agropecuario José Rodríguez Labandera-Vargas Flores, Mildred MarleneUniversidad Regional Autónoma de los Andes- Maestría en Gestión de la Educa- 
ción-2013.

- Sistema de acción para potenciar la investigación formativa en la carrera de alimentos de la Universidad Técnica Estatal de Quevedo-Llerena Guevara, Teresa-Universidad Regional Autónoma de los Andes- Maestría en Gestión de la Educación-2013.

c) Cuatro artículos científicos:

- La investigación formativa de pregrado en la Universidad Católica de Cuenca. Los Semilleros de Investigación-Vanegas Quizhpi, Oscar Santiago; Curay Banegas, Edgar Rigoberto; García Guevara, Wilson- YACHANA Revista Científica, vol. 6, núm. 2 (noviembre de 2017), pp. 76-83

- El proceso formativo en investigación: un estudio de caso en Ecuador-Val, Pablo del, Suárez, Rafael, Sánchez, Francisco, Bravo-Sánchez, Santiago - Pontificia Universidad Católica del Ecuador- Santo Domingo- 2017

- Límites y ventajas en el proceso de enseñanza y aprendizaje de la investigación formativa-Ruano Ibarra, Luis Eduardo-Universidad San Gregorio de Portoviejo-Revista San Gregorio, 2017, no.16, edición especial, junio, (142-153), ISSN 2528-7907

- El proceso de enseñanza-aprendizaje y la investigación formativa, una relación necesaria en la universidad del siglo XXI-Menéndez Padrón, Adalberto; Pérez Mato, Daisy de la Caridad-Universidad Laica Vicente Rocafuerte- YACHANA Revista Científica, vol. 5, núm. 3 (noviembre de 2016), pp. 70-75- 2018.

d) Artículos científicos:

- Scopus. Ocho artículos, dos en español y seis en inglés, a saber:

- Cómo los docentes no universitarios perciben la investigación educativa.

- Las disposiciones subjetivas de los docentes en la superación de las resistencias al cambio ante procesos cíclicos de formación basados en la investigación.

o Evaluation of a pedagogical model for student engagement in learning activities.

o Learning from each other. The design and implementation of a crosscultural research and professional development model in Italian and U.S. toddler classrooms

o Mentoring partnerships in science education.

o Teachers use of research evidence in practice. a pilot study of feedback to enhance learning.

o Understanding student behavioral engagement. Importance of student interaction with peers and teachers.

o Variables affecting positive faculty-student relationships in an ecuadorian university.

- SciELO. Diez artículos en español, a saber:

- Abrir las aulas. el vínculo entre docencia, investigación y vinculación comunitaria.

- Aportes a la formación del profesorado constructivista. Resultados en dos países. 
- Causales de deserción en equipos de investigación formativa.

- Desarrollo profesional en educadoras de párvulos. análisis de una experiencia formativa desde la investigación-acción y la interacción mediada.

o Experiencias de formación como investigadores educativos de estudiantes de un programa de doctorado en educación.

o Impacto en la producción científica de un programa experiencial de preparación para la investigación dirigido a docentes universitarios.

- La formación docente universitaria. claves formativas de universidades españolas.

o La investigación formativa y la formación para la investigación en el pregrado.

- Mirada a las investigaciones sobre formación investigativa en la universidad latinoamericana. Estado del arte 2010-2017.

o Tutoría académica. Otra forma de enseñanza.

- Dialnet. Un artículo, a saber:

o La investigación formativa en los procesos de investigación asumidos.

- DOAJ. 30 artículos, a saber:

o El papel de la investigación en la formación universitaria.

- Enseñanza de la investigación y epistemología de los docentes.

o Estudio etnográfico en docentes del programa nacional de formación en mecánica.

- Experiencias de formación como investigadores educativos de estudiantes de un programa de doctorado en educación.

o Formación de investigadores de las ciencias sociales y humanas en el enfoque fenomenológico hermenéutico (de Van Manen) en el contexto hispanoamericano.

- Formación investigativa en estudiantes de pregrado mediante entornos de aprendizaje móvil con APPS.

- Hacia un desarrollo de las historias personales y profesionales de los docentes.

o Influencia que ejerce sobre la formación integral de los estudiantes la realización de trabajos investigativos desde el primer año de la carrera. Pedagogía Universitaria.

o Interacción estudiante-investigador. Relación pedagógica y profesional que sustenta la formación en el postgrado.

o Investigación educativa o investigación pedagógica. El caso de la investigación en el Distrito Capital.

o Investigación formativa e investigación productiva de conocimiento en la universidad.

o Investigación formativa y cultura científica en las facultades de educación de Nariño. 
o La formación docente autogestionada a través de la investigación-acción.

- La formación investigativa en la educación superior en América Latina y el Caribe. una aproximación a su estado del arte.

o La investigación formativa en ambientes ubicuos y virtuales en Educación Superior.

o La investigación formativa en la actividad curricular.

o La investigación formativa en la universidad ecuatoriana. Una experiencia personal.

o La investigación formativa en los procesos de investigación asumidos en la universidad.

- La investigación formativa. Una experiencia del cuerpo académico estudios en educación.

- Los artículos científicos como herramienta de aprendizaje en las escuelas de arquitectura.

- Reconocimiento y desarrollo profesional docente. Una experiencia con docentes que participan de programas de especialización para profesores de educación básica.

o Semilleros de investigación. una estrategia para la formación de investigadores.

o Supervisors' Perceptions of Research Competencies in the Final Year Project.

o Trabajo científico-estudiantil. Historia y proyección. El caso de una facultad de la Universidad de Guayaquil.

- Redalyc. 12 artículos, a saber:

o Aula expandida. Una experiencia de investigación formativa.

o Discursos, prácticas y perspectivas de los formadores de docentes.

o Docencia e investigación. Vínculo en construcción.

o Generación XX. teorías sobre la juventud en la era contemporánea.

o Imaginación, herramientas cognitivas y alumnos renuentes.

o Investigación curricular y desarrollo profesional.

o Investigación formativa e investigación productiva de conocimiento en la universidad.

o La imaginación. una olvidada caja de herramientas del aprendizaje.

o La investigación acción. Una herramienta metodológica heurística para la comprensión y transformación de realidades y prácticas y socioeducativas.

o La investigación formativa y la investigación basada en el diseño. Dos perspectivas de alcance.

o La vinculación docencia investigación como estrategia pedagógica.

o Programa de desarrollo de la investigación educativa. 
e) tres tesis de doctorado:

- Formación de la competencia investigativa en docentes de la Educación Media Superior.

- La competencia pedagógica investigativa en la licenciatura biología-geografía.

- La formación de la competencia investigativa en los estudiantes de licenciatura en Ciencias de la Educación, menciones fisicomatemáticas

f) Tres libros:

- La investigación educativa en México y Brasil.

- Investigación social desde la práctica educativa.

- Vínculo docencia-investigación para una formación integral.

\section{DISCUSIÓN Y CONCLUSIONES}

Con el apoyo de los programas Mendeley y Endnote, se identificó los autores con más citaciones, cuyos artículos fueron leídos para seleccionar los que más aportan al tema de estudio. Entre los principales hallazgos, se determinó que:

- Con la implementación de la investigación formativa, tanto docentes como estudiantes participan en el trabajo académico. Esta participación les permite, sobre todo a los estudiantes, adquirir conocimientos investigativos y una representación cultural y científica del mundo.

- Motiva a los estudiantes a comprender y aspirar a los valores del investigador, tales como: objetividad, respeto a la evidencia, honestidad, rigor académico, entre otros. Asimismo, se gesta una variante de aprendizaje a través de la comunicación oral, aspecto que prepara a los aprendientes para el desarrollo de las competencias comunicativas.

- Los educandos se comprometen con la ejecución de actividades más productivas que les permiten el desarrollo de procesos cognitivos superiores. Desde la necesaria comprensión, el análisis, la síntesis, la resolución de problemas, la toma de decisiones, la evaluación y la creación de contenidos y procedimientos. Además, de comprometer a los estudiantes en procesos de aprendizaje colaborativo, se promueve la interacción entre estudiantes y docente.

- Emprender procesos de investigación formativa, posibilitará que la investigación pueda ser vista por los actores educativos como una herramienta que proporciona rigor académico, pero que no constituye una verdad sagrada o dogma.

- El desarrollo y puesta en práctica en las aulas de procesos didácticos sustentados en la investigación, también prepara a estudiantes y docentes para que asuman el criterio de ciencia provisional, muy adecuada en la era postmoderna. Con ello se genera explicaciones y formulaciones teóricas con fundamento y evidencia científica, en el marco de una hipótesis en continua revisión.

- Apoya a los actores educativos en el conocimiento, preparación y empleo de estrategias didácticas e investigativas, como también al desarrollo de procesos de investigación guiada y autónoma. Con ello, se fortalece, además de la producción científica en el estudiantado, el pensamiento crítico y dialógico, a través de la estructuración de ideas y 
criterios sustentados tanto en los razonamientos y justificaciones académicas como en la experiencia acumulada por comunidades científicas.

En tanto el análisis y discusión constituyen el centro de la actividad académica, prepara a los estudiantes en la formulación de argumentos y enunciados que puedan demostrarse. De igual manera, les ayuda a respetar puntos de vista diferentes a los suyos.

\section{REFERENCIAS}

Aguilera-Morales, D., Martín-Páez, T., Valdivia-Rodríguez, V., Ruíz-Delgado, Á., Williams-Pinto, L., Vílchez-González, J., \& Perales-Palacios, F. (2018). La enseñanza de las ciencias basada en indagación. Una revisión sistemática de la producción española. Revista de Educación $N^{\circ} 381$. http://bit.ly/3bOrCCh

Blessinger, P. y Carfora, J. (Ed.). (2014). Inquiry-based learning for the arts, humanities and social sciences. A conceptual a practical resource for educators. Emerald. http://ebookcentral.proquest.com

Gough, D., Oliver, S., Thomas, J. (2012). An introduction to systematic reviews. SAGE.

Gómez-Luna, E., Fernando-Navas, D., Aponte-Mayor, G. y Betancourt-Buitrago, L. A. (2014). Metodología para la revisión bibliográfica y la gestión de información de temas científicos, a través de su estructuración y sistematización. DYNA, 81(184), 158-163. http://bit.ly/3bPzK5F

Hernández, F., Forés, A., Sancho, J.M., Sánchez, J.A., Casablancas, S., Creus, A., Herraiz, F., \& Padró, C. (2011). Aprender desde la indagación en la universidad. Octaedro

Oropeza, M. (2016). Formación de la competencia investigativa en docentes de la educación media superior. Tesis de doctorado, Universidad de La Habana, La Habana, Cuba. http://ebookcentral.proquest.com

Ronquillo, L. (2017). La formación de la competencia investigativa en los estudiantes de licenciatura en ciencias de la educación, menciones fisicomatemáticas. Tesis de doctorado, Universidad de La Habana, La Habana, Cuba. http://ebookcentral. proquest.com

Ruíz del Castillo, A. y Rojas-Soriano, R. (2006). Vínculo docencia-investigación para una formación integral. Plaza y Valdés.

Torres, A. (s.f.). Aprendizaje basado en la investigación. Técnicas didácticas. TEC de Monterrey. http://bit.ly/2woaZx5

Torres-Verdugo, Á. (2016). Guía de fuentes de información iberoamericana para la investigación educativa. http://bit.ly/2SH34DT

UNAM. (2015). Vocabulario controlado. Iresie. http://bit.ly/2HAc6w9 